\title{
The Impact of the Spectral Filter Bandwidth on the Spectral Entanglement and Indistinguishability of Photon Pairs of SPDC Process
}

\author{
Ali Mohammadi, Atoosa Sadat Arabanian*, and Ali Dalafi \\ Laser and Plasma Research Institute, Shahid Beheshti University, Tehran, Iran \\ *Courresponding author's email: a_arabanian@sbu.ac.ir
}

Received: Jun. 10, 2017, Revised: Jul. 2, 2017, Accepted: Jul. 27, 2017, Available Online: Dec. 1, 2018

DOI: 10.29252/ijop.12.2.119

\begin{abstract}
In this paper, we have investigated the dependence of the spectral entanglement and indistinguishability of photon pairs produced by the spontaneous parametric downconversion (SPDC) procedure on the bandwidth of spectral filters used in the detection setup. The SPDC is a three-wave mixing process which occurs in a nonlinear crystal and generates entangled photon pairs and utilizes as one of the most useful resources in a variety of fields such as quantum computation, quantum cryptography, and quantum communication. The amount of the spectral entanglement and the indistinguishability of photon pairs are the two critical characteristics of the photon pairs determining their potential applications. The degree of the spectral entanglement of a quantum system, i.e. photon pairs, is determined by the entanglement entropy which is a measure of the system disorder. First, we derive the eigenvalue equation of reduced density operator of the signal and the idler photons in terms of the bandwidth of spectral filters. Then, by numerically solving the eigenvalue equation, we calculate the Schmidt coefficients for different values of the bandwidth of spectral filters. Finally, by calculating the entropy operator one can obtain the dependence of spectral entanglement of the photon pairs on filter bandwidth. The amount of indistinguishability of the photon pairs is measured by the visibility of the HOM diagram. Since the whole spectral information of the photon pairs exists in their two-photon mode function, using this function and the presented model we calculate the amount of indistinguishability by obtaining the visibility of the HOM diagram for different values of the bandwidth of the spectral filters. In this way,
\end{abstract}

the dependence of the indistinguishability of the photon pairs on this quantity is reported.

KeYwORDS: Spontaneous Parametric DownConversion, Entanglement, Entangled Photon Pairs, Single Photon sources, Indistinguishability, entropy, reduced density operator.

\section{I.INTRODUCTION}

Entanglement is one of the most interesting and fascinating concepts in the quantum optics field. Today, the possibility of producing the entangled quantum states has been experimentally provided. In practice, the entangled photons can be generated by different methods such as the cascade emission of atoms and the spontaneous parametric down conversion (SPDC), etc. [1]. In a nonlinear crystal, due to the second-order nonlinear interaction, a small fraction of the pump laser photons can decay into the photon pairs by the process of spontaneous parametric down conversion. These two photons are generated simultaneously and follow the laws of energy and momentum conservation. In 1970, the SPDC was introduced for the first time, as a method to generate entangled states of light [2]. From that time, a tremendous amount of experiments has been done in order to generate entangled photon pairs using the SPDC procedure [3]. Investigations of the correlated properties of entangled two-photon states have attracted much attention in quantum optics and information fields, such as quantum imaging, quantum cryptography and quantum 
teleportation. The photons generated by the SPDC can be entangled in different degrees of freedom such as polarization, frequency, angular momentum, etc. The entanglement of two photons in a particular quantity means that the two-photon mode function cannot be decomposed into two individual functions in the space of that quantity.

Since measurement and analysis of polarization entanglement of photon pairs is often much easier due to the availability of efficient polarization-control elements, Therefore, photon pairs entangled in polarization are most widely used in many applications of quantum optics. The SPDC process based on type II phase matching is a standard resource for generation of the polarization entangled photon pairs. Although type-I phase matched SPDC can be also used for this aim, but, the type-II SPDC process can provide a richer range of entanglement options than those of type-I SPDC and it creates a more flexible, and reliable quantum apparatus for many applications of quantum optics such as cryptographic applications. Other significant differences between the type-I and type-II SPDC are spectral and spatial emission properties of the photons. For example, type-II SPDC has significantly narrower spectral emission than that of type-I SPDC [4]. Furthermore, unlike type-I SPDC which has a ring-shape transverse emission pattern due to the degenerate nature of the polarization state of the photon pair, type-II SPDC emission pattern forms two rings, one belonging to the ordinary ray and the other to the extraordinary ray [4].

For photon pairs generated by type II SPDC, the degree of the spectral entanglement and the indistinguishability are the two critical characteristics [5]. The spectral entanglement of photon pairs which corresponds to a correlation between frequency modes of signal and idler photons is considered as a destructive factor for polarization-entangled photon pairs generated by type II SPDC process so that researchers looks for a way to reduce this entanglement [6]. For example, in quantum communication the existence of spectral entanglement leads to a disturbance in data transformation and undesired distinguishing information [7]. For this reason, the researchers are always looking for an experimental solution for eliminating these unwanted entanglements [6]. The other important property of the photon pairs is their amount of indistinguishability. This property states that how similar two different photons of signal and idler are. The amount of indistinguishability is measured by the visibility of the Hong-Ou-Mandel (HOM) diagram [8]. One of the experimental methods for decreasing of spectral entanglement and increasing of indistinguishability of photon pairs is the use of spectral filters just before detectors in the detection setup. In this paper, the impact of the spectral filter bandwidth on the two above-mentioned properties of photon pairs is investigated.

\section{THEORY}

The SPDC procedure is a three-wave interaction which occurs in the birefringent crystal medium. The birefringent crystal is pumped by a laser with a suitable polarization based on the phase matching conditions. During the laser-crystal interaction, some of the pump photons are converted to the entangled signal and idler photons. The frequency of generated photons is less than that of the pump photons. In this procedure, the frequency and momentum of the signal and idler photon pairs are such that the energy and momentum conservation laws are satisfied. The momentum conservation relation is sometimes called the phase matching condition.

There are two phase matching types for the SPDC process. In the first case, known as type-I phase matching, the polarization of the signal and idler photons are parallel and perpendicular to the polarization of the pump beam. In the latter case which is called the type-II phase matching, the signal and idler photons have perpendicular polarization, and the polarization of one of them is parallel to the polarization of the pump. Furthermore, the SPDC process is divided into collinear and non-collinear geometries [4]. In the collinear geometry, the propagation directions of pump, 
signal and idler photons are parallel to each other while in the non-collinear geometry, the propagation directions of the signal and idler photons make an angle with pump direction (Fig. 1). In this paper, calculations for the type-II SPDC process with collinear geometry which are most widely used in many applications of quantum optics have been performed. Of course, similar calculations can be done for type-I SPDC process and for noncollinear geometry.
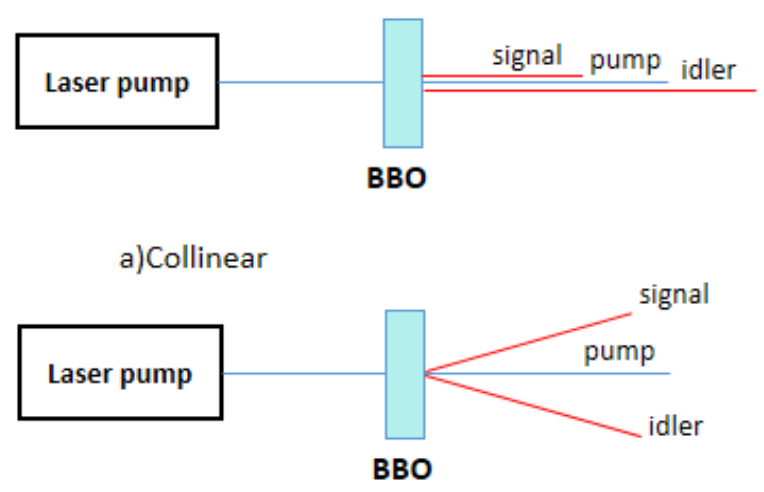

b)Noncollinear

Fig. 1. (a) Collinear and (b) noncollinear geometries of SPDC procedure.

The efficiency of the SPDC procedure depends on the input pump intensity, the type of the crystal, and the phase matching conditions.

The energy conservation and the phase matching relations are, respectively, given by the following relations [9]

$$
\begin{aligned}
& \hbar \omega_{p}=\hbar \omega_{s}+\hbar \omega_{i} \\
& \hbar \mathbf{k}_{p}=\hbar \mathbf{k}_{s}+\hbar \mathbf{k}_{i}
\end{aligned}
$$

The spectral properties of photon pairs of the SPDC procedure are determined by the pure state $\rho=|\psi\rangle\langle\psi|$ where the state vector $|\psi\rangle$ is given by [10]:

$$
|\psi\rangle=\int d \Omega_{s} d \Omega_{i} \phi\left(\Omega_{s}, \Omega_{i}\right) \hat{a}^{+}\left(\Omega_{s}\right) \hat{a}^{+}\left(\Omega_{i}\right)|0,0\rangle
$$

where $\Omega_{n}=\omega_{n}-\omega_{n 0}$ with $n=s, i$ where $\omega_{n}$ is the frequency of the signal or idler photons and $\Omega_{n}$ is the deviation from the central frequency $\omega_{n} 0$. $\hat{a}^{+}\left(\Omega_{n}\right)$ is the creation operator of single photons of signal or idler. The photons generated by the SPDC process are considered as wave packets with specified spectral width. All information about spectral distribution of the photon pairs is described by the normalized mode function $\varphi\left(\Omega_{s}, \Omega_{i}\right)$. We consider a BBO crystal with length $L$ which is pumped by a pulsed laser having a Gaussian spectral distribution with width $\sigma_{p}$. The corresponding mode function of the photon pairs is given by [10]:

$$
\begin{aligned}
\varphi\left(\Omega_{s}, \Omega_{i}\right) & =N \exp \left[-\frac{\left(\Omega_{s}+\Omega_{i}\right)^{2}}{4 \sigma_{p}^{2}}\right] \sin c\left[\frac{\Delta_{k} L}{2}\right] \\
& \times \exp \left[-i \frac{\Delta_{k} L}{2}\right] \exp \left[-\frac{\Omega_{s}^{2}}{4 \sigma_{s}^{2}}\right] \exp \left[-\frac{\Omega_{i}^{2}}{4 \sigma_{i}^{2}}\right]
\end{aligned}
$$

where $N$ is the normalization coefficient and the first exponential function signifies the pump laser spectral distribution as a Gaussian curve. The characteristics of the nonlinear crystals such as the thickness, cut angles and its Sellmeier equations have been included in the calculations by the phase mismatching relation of $\Delta_{\mathrm{k}}=k_{p}-k_{s}-k_{i}$ whose first order Taylor series approximation gives us $\Delta_{\mathrm{k}}=\left(N_{p^{-}}\right.$ $\left.N_{s}\right) \Omega_{\mathrm{s}}+\left(N_{p}-N_{i}\right) \Omega_{\mathrm{i}}$ where $N_{p}, N_{s}$ and $N_{i}$ are the inverse of group velocities of pump, signal and idler beams, respectively. Finally, the last two exponential functions in Eq. (4) determine the spectral distribution functions of the spectral filters used in the detection setup of the photon pairs which have been modeled by Gaussian functions with bandwidths $\sigma_{\mathrm{s}}$ and $\sigma_{\mathrm{i}}$.

In Fig. 2 we have schematically shown the generation and detection setup of the entangled photon pairs of the SPDC process with collinear and non-collinear geometries. 

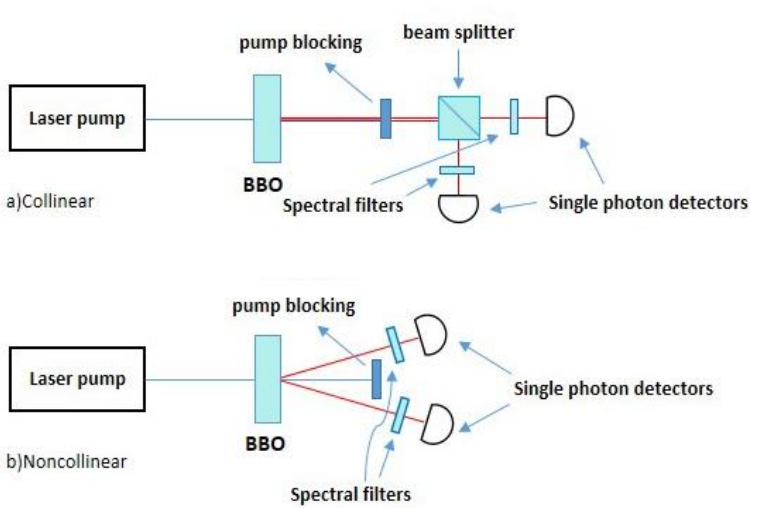

Fig. 2. The generation and detection setups of the entangled photon pairs of the SPDC process for geometries of collinear (a) and noncollinear (b).

The reduced density operator of the signal (idler) photon is derived by taking a trace of the density operator over the degrees of freedom of the idler (signal) photon which is given by:

$\rho_{s}=\operatorname{Tr}[|\psi\rangle\langle\psi|]_{i}=$

$\int d \Omega_{s} d \Omega_{s}^{\prime} \phi\left(\Omega_{s}, \Omega_{s}^{\prime}\right) \hat{a}^{+}\left(\Omega_{s}\right)|0\rangle\langle 0| \hat{a}\left(\Omega_{s}^{\prime}\right)$

where

$$
\phi\left(\Omega_{s}, \Omega_{s}^{\prime}\right)=\int d \Omega_{i} \phi\left(\Omega_{s}, \Omega_{i}\right) \phi\left(\Omega_{s}^{\prime}, \Omega_{i}\right)
$$

is the signal photons mode function.

\section{A. Spectral Entanglement of Photon Pairs}

The spectral entanglement between the signal and idler photons indicates a correlation between a single frequency mode of signal (idler) photon and several frequency modes of the idler (signal) [6]. For this purpose, one can use the joint spectral intensity (JSI) function which is defined by the square of product of phase matching terms multiplied by pump laser curve in Eq. (4) as follows [9]:

$$
J S I=\left|\exp \left[-\frac{\left(\Omega_{s}+\Omega_{i}\right)^{2}}{4 \sigma_{p}^{2}}\right] \sin c\left[\frac{\Delta_{k} L}{2}\right] \exp \left[-i \frac{\Delta_{k} L}{2}\right]\right|^{2}
$$

This function is the probability of the signal photon having frequency $\omega_{s}$ and the idler photon having frequency $\omega_{i}$.

One of the criteria for measuring the amount of spectral entanglement between the two parts of a bipartite quantum system is determined by the amount of separability of its subsystems states [11]. In the following equations we have given two examples for the total state of photon pairs when its subsystems (signal and idler) are uncorrelated (Eq. (8)) and when they are correlated (Eq. (9)):

$$
\begin{aligned}
& \rho=\rho_{s} \otimes \rho_{i} \\
& \rho \neq \rho_{s} \otimes \rho_{i}
\end{aligned}
$$

It has been proved that for bipartite systems with a pure state, the amount of state separability is obtained by calculating the Schmidt coefficients and the entanglement entropy of one of the subsystems reduced density operator as follows [11]:

$$
E(\rho)=s\left(\rho_{s}\right)=\sum_{m} \lambda_{m} \quad(m=1,2, \ldots)
$$

The entanglement entropy $E(\rho)$ determines the amount of inseparability of the total state $\rho$. The Schmidt coefficients $\lambda_{\mathrm{m}}$ which are the common real eigenvalues of the signal and idler reduced density operators can be obtained by solving the following integral eigenvalue equation numerically [6]:

$$
\int d \Omega_{s}^{\prime} K\left(\Omega_{s}, \Omega_{s}^{\prime}\right) \Gamma_{m}\left(\Omega_{s}^{\prime}\right)=\lambda_{m} \Gamma_{m}\left(\Omega_{s}\right)
$$

The kernel function $K\left(\Omega_{s}, \Omega_{s}^{\prime}\right)$ in Eq. (11) is the signal single photon mode function, i.e., Eq. (6) and the functions $\Gamma_{m}\left(\Omega_{s}\right)$ are the eigenfunctions of $\rho_{\mathrm{s}}$. For numerically solving Eq. (11) and obtaining the Schmidt coefficients, we discretize the integral and use the Gaussian quadrature method [12]. In this way, Eq. (11) can be written as the following matrix form:

$$
\begin{aligned}
& \left(\begin{array}{c}
\lambda_{1} \Gamma_{1}\left(\Omega_{s}\right) \\
\lambda_{2} \Gamma_{2}\left(\Omega_{s}\right) \\
\vdots
\end{array}\right)= \\
& \left(\begin{array}{ccc}
K\left(\Omega_{s 1}, \Omega_{s 1}^{\prime}\right) & K\left(\Omega_{s 1}, \Omega_{s 2}^{\prime}\right) & \ldots \\
K\left(\Omega_{s 2}, \Omega_{s 1}^{\prime}\right) & K\left(\Omega_{s 2}, \Omega_{s 2}^{\prime}\right) & \ldots \\
\vdots & \vdots & \ddots
\end{array}\right)\left(\begin{array}{c}
\Gamma_{1}\left(\Omega_{s}\right) \\
\Gamma_{2}\left(\Omega_{s}\right) \\
\vdots
\end{array}\right)
\end{aligned}
$$


By calculating the numerical values of the kernel matrix elements in Eq. (12) and its eigenvalues, the Schmidt coefficients $\lambda \mathrm{m}$ will be obtained.

\section{B. Spectral Indistinguishability of Photon Pairs}

The indistinguishability of two photons indicates the amount of the similarity of their states and also quantifies the overlap between their describing states. In the case of complete overlap of states, the amount of indistinguishability is considered as the maximum value while with no overlap the amount of indistinguishability is considered to be zero. The most famous experimental method for evaluation of indistinguishability of photon pairs is through the investigation of their interference in the HOM interferometer. As has been shown in Fig. 3 in the HOM interferometer when the two indistinguishable photons enter simultaneously form the two beam splitter inputs and they simultaneously exit from an output of the beam splitter.

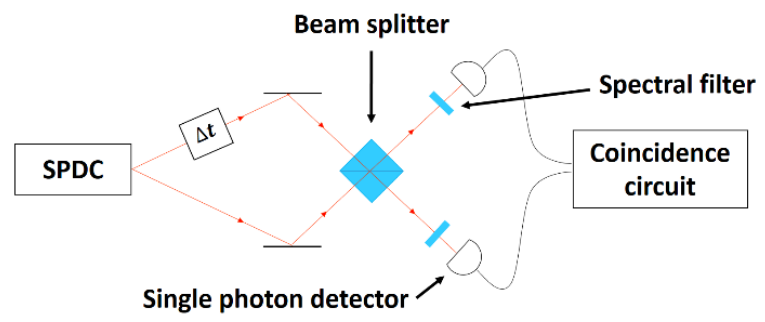

Fig. 3. The HOM interferometer

The more amount of the indistinguishability of photons leads the less probability of their simultaneous observation in the beam splitter outputs which leads to the well-known HOM valley plot [13].

If the temporal resolution of the detector is longer than the coherence time of the photons, the probability of having a coincidence between the outputs of the beam splitter is given by the integral over the detection times of the second-order coherence function:

$G^{(2)}\left(t_{1}, t_{2}\right)=\left\langle\hat{E}_{1}^{(-)}\left(t_{1}\right) \hat{E}_{2}^{(-)}\left(t_{2}\right) \hat{E}_{1}^{(+)}\left(t_{1}\right) \hat{E}_{2}^{(+)}\left(t_{2}\right)\right\rangle$

which is given by [7]:
$R_{c c}=\int d t_{1} d t_{2} G^{(2)}\left(t_{1}, t_{2}\right)$

In Eq. (13), $\hat{E}_{1}$ and $\hat{E}_{2}$ are the field operators and $t_{1}$ and $t_{2}$ are the detection times in the detectors 1 and 2 [10]. Calculations show that the coincidence probability for the photon detection in the two detectors is as follows:

$R_{c c}=1-I(\Delta t)$

It is given in terms of the delay time

$\Delta t=t_{2}-t_{1}$, and:

$I(\Delta t)=\frac{\int d \Omega_{s} d \Omega_{i} \phi\left(\Omega_{s}, \Omega_{i}\right) \phi^{*}\left(\Omega_{i}, \Omega_{s}\right) e^{-i\left(\Omega_{s}-\Omega_{i}\right) \Delta t}}{\int d \Omega_{s} d \Omega_{i} \phi\left(\Omega_{s}, \Omega_{i}\right) \phi^{*}\left(\Omega_{s}, \Omega_{i}\right)}$

As is seen from Eq. (16) the function $\mathrm{I}(\Delta \mathrm{t})$ is in terms of the product of $\phi\left(\Omega_{s}, \Omega_{i}\right)$ multiplied by $\phi^{*}\left(\Omega_{i}, \Omega_{s}\right)$. The larger amount of this product causes to the larger amount of $\mathrm{I}(\Delta \mathrm{t})$ (with the assumption of fixed $\Delta t$ ). This product is maximum when the two functions have the same form of dependence on the variables $\Omega_{s}, \Omega_{i}$. In other words, when the two functions are similar. The closer value of $\Omega_{s}$ to $\Omega_{i}$ leads to the more similar two functions $\phi\left(\Omega_{s}, \Omega_{i}\right)$ and $\phi^{*}\left(\Omega_{i}, \Omega_{s}\right)$ to each other and therefore the lager amount of their product. That is why the value of I $(\Delta t=0)$ is considered as the amount of spectral indistinguishability between the signal and idler photons [10]. Based on Eq. (15) the more indistinguishable the two photons results to the smaller coincidence probability (the minimum of HOM plot). Because of this reason, in most experiments the visibility of the HOM dip is considered as a criterion for the measurement of the photon pair's indistinguishability. The visibility of the HOM dip plot is defined as [10]:

$V=\frac{\max \left(R_{c c}(\Delta t)\right)-\min \left(R_{c c}(\Delta t)\right)}{\max \left(R_{c c}(\Delta t)\right)+\min \left(R_{c c}(\Delta t)\right)}$

\section{III.RESULTS AND DISCUSSION}

In order to investigate the impact of spectral filters bandwidth used in the detection setup 
on the spectral entanglement of photon pairs, we calculate the spectral entanglement for different values of spectral filters bandwidth.

Our calculations are based on the following feasible experimental data. We consider a laser pump with wavelength of $800 \mathrm{~nm}$ and with the spectral bandwidth of $12 \mathrm{~nm}$. It is also considered a type II phase matching BBO crystal with a length of $2.1 \mathrm{~nm}$ for a degenerate SPDC procedure in which $\lambda_{s}=\lambda_{i}=2 \lambda_{p}=1600 \mathrm{~nm}$. Our calculations have been performed for the type-II SPDC process with collinear geometry.

Based on these data, we calculate the Schmidt coefficients for different values of spectral filter bandwidth in three different detection setup (both signal and idler beams filtering, signal beam filtering, idler beam filtering) and obtain the entanglement entropy. The dependence of the entanglement entropy on the spectral filters bandwidth can be obtained by Eq. (11) which has been shown in Fig. 4 for a type II SPDC procedure. As is observed from Fig. 4, the filtering process can cause the reduction of spectral entanglement. The narrower filter bandwidths lead to the smaller spectral entanglement of photon pairs. In Fig. 5 the JSI function of photon pairs generated by the SPDC has been plotted for different filtering setups. As shown in Fig. 5, by considering each signal (idler) frequency mode and plotting a line parallel to the idler (signal) axis, the spectral range of the idler (signal) correlated with that frequency mode and the probability of the correlation between different modes are determined. In a filtering process, some of the photons with frequencies far away from the central frequency are blocked. In this way, each frequency mode of the signal (idler) photons are less correlated to the other idler (signal) frequency modes.

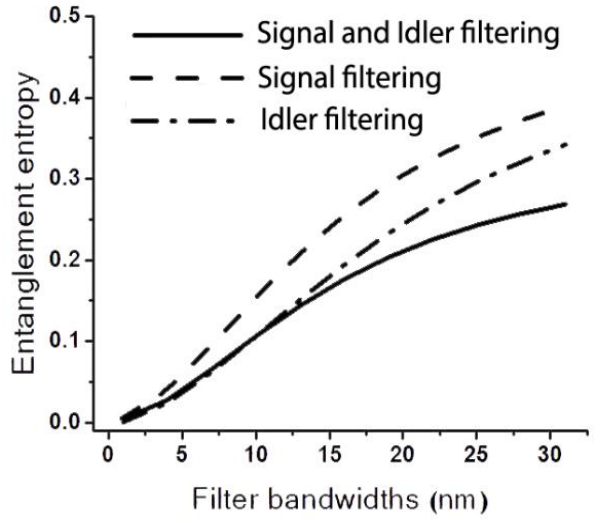

Fig. 4. The entanglement entropy of the signal-idler photon pairs vs. filter bandwidth in the type II phase matching SPDC procedure in three different filtering setups.

In fact, spectral filtering of the signal and idler photons leads to the increase of spectral information and decrease of spectral entanglement.
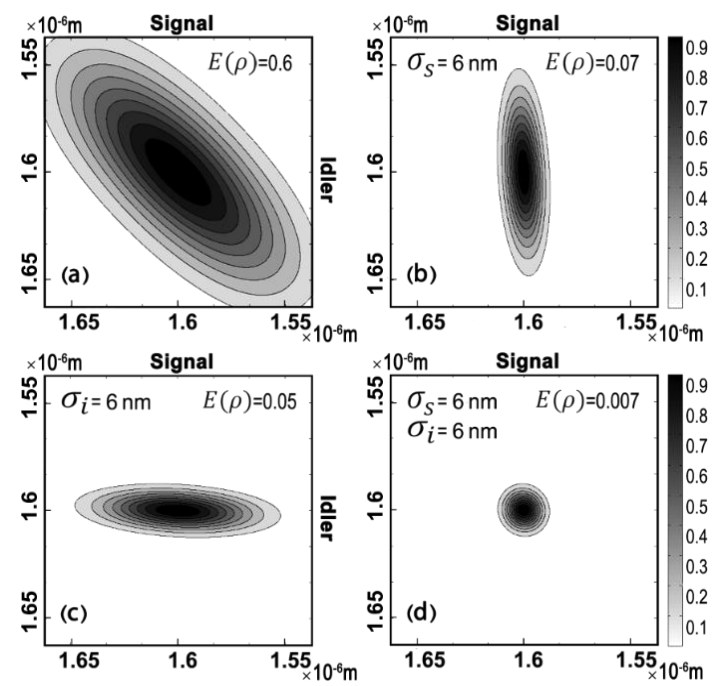

Fig. 5. The JSI function of photon pairs generated by the degenerate SPDC for a pump laser with central frequency of $800 \mathrm{~nm}$ and a spectral filter bandwidth of $12 \mathrm{~nm}$ for different filtering setups: (a) without filtering, (b) signal filtering, (c) idler filtering (d) signal and idler filtering. 


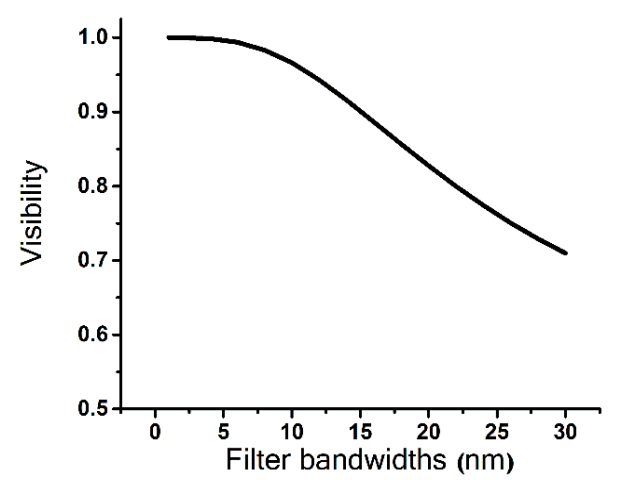

Fig. 6. The visibility of photon pairs generated by the SPDC process versus the bandwidth of spectral filters.

In order to investigate the impact of spectral bandwidth of the filters used in the detection setup on the amount of spectral indistinguishability of photon pairs, we have plotted in Fig. the visibility of the HOM dip versus the filters bandwidths. Here, we have considered a BBO crystal with length $10 \mathrm{~mm}$ in a type-II SPDC process which is pumped with a laser with properties expressed in the previous section.

As is seen from Fig. 6, for spectral filters with small bandwidths, the visibility of the HOP dip plot gets larger and therefore the indistinguishability of photon pairs of the SPDC output is increased. In fact, the filtering process causes just those signal and idler photons whose frequencies are inside the bandwidth of the spectral filters to reach to the detectors and the others are blocked. It leads to the increase of spectral indistinguishability of photon pair's.
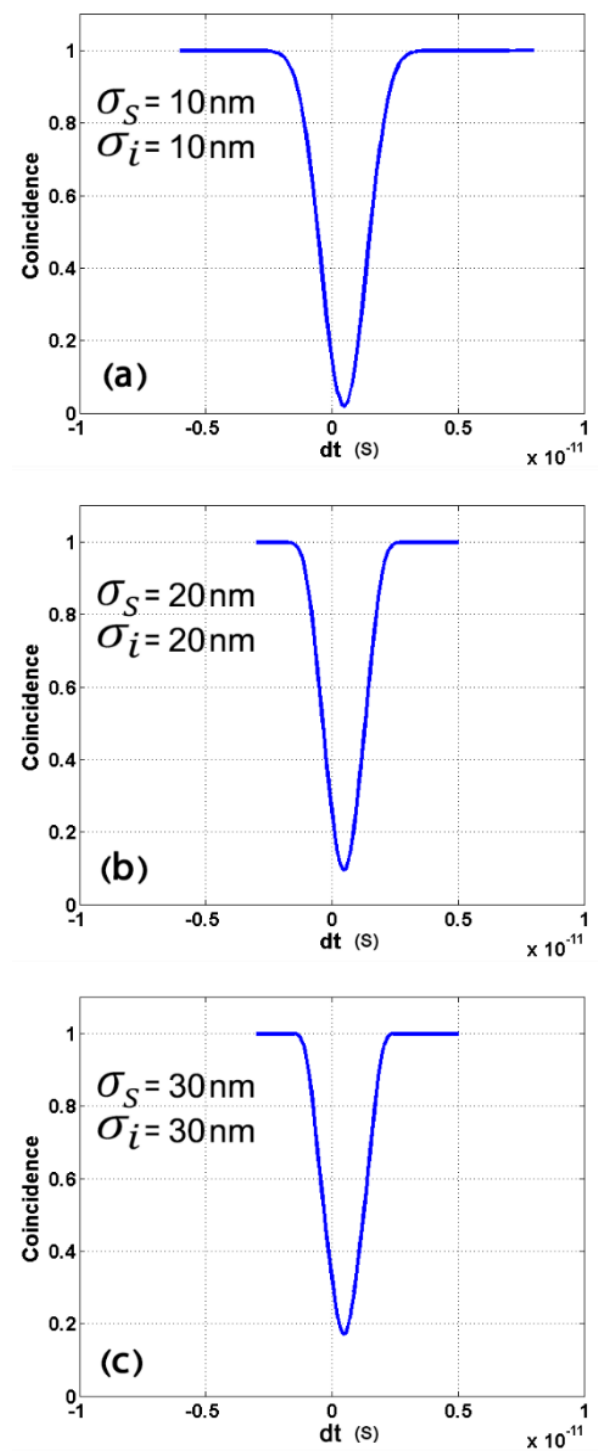

Fig. 7. The HOM dip plot for photon pairs generated by the SPDC for three different bandwidths of spectral filters.

In Fig. 7 the coincidence rate of simultaneous detection of the photon pairs in the two outputs of the beam splitter has been plotted versus the optical path difference of the two photons.

In Figs.7a-7c the HOM dip plots have been shown for three different bandwidth of spectral filters. As is seen from Fig. 7 the smaller bandwidth of spectral filters leads to the smaller value of the HOM dip which corresponds to the lager values of the HOM dip visibility and also the larger indistinguishability of photon pairs. 


\section{IV.CONCLUSION}

In this paper, we have investigated how the spectral entanglement and indistinguishability of the photon pairs generated by the type II SPDC process depend on the bandwidth of the spectral filters used in the detection setup.

Our calculations show that the spectral entanglement of photon pairs decreases with the reduction of the spectral filters bandwidth. Based on our simulations, for those applications of entangled photon pairs where the spectral entanglement is considered as a destructive factor, one can decrease the spectral entanglement through a suitable filtering. On the other hand, it was observed that the indistinguishability of photon pairs is decreased by decreasing the bandwidth of the spectral filters. In this way, it can be concluded that in the interferometry based applications where the high value of photon pair's indistinguishability is considered as a vital factor, it can be increased by using suitable spectral filters.

\section{REFERENCES}

[1] S. Takeuchi, "Recent progress in singlephoton and entangled-photon generation and applications," Jpn. J. Appl. Phys, Vol. 53, pp. 030101(1-11), 2014.

[2] D. C. Burnham and D. L. Weinberg, "Observation of simultaneity in parametric production of optical photon pairs," Phys. Rev. Lett, Vol. 25, pp. 84-87, 1970.

[3] J. Yin, J-G. Ren, H. Lu, Y. Cao, H-L. Yong, Y-P. Wu, Ch. Liu, Sh-K. Liao, F. Zhou, Y. Jiang, X-D. Cai, P. Xu, G-Sh. Pan, J-J. Jia, YM. Huang, H. Yin, J-Y. Wang, Y-A. Chen, Ch-Z. Peng, and J-W. Pan, "Quantum teleportation and entanglement distribution over 100-kilometre free-space channels," Nature, Vol. 488, pp. 185-188, 2012.

[4] O. Kwon, Y. W. Cho, and Y. H. Kim, "Single-mode coupling efficiencies of type-II spontaneous parametric down-conversion: Collinear, noncollinear, and beamlike phase matching," Phys. Rev. A, Vol. 78, pp. 053825 (1-4), 2008.
[5] R. Quan, M. Wang, F. Hou, Zh. Tai, T. Liu, Sh. Zhang, and R. Dong, "Characterization of frequency entanglement under extended phase matching conditions," Appl. Phys. B, Vol. 118, pp. 431-437, 2015.

[6] W. P. Grice, A. B. U'ren, and I. A. Walmsley, "Eliminating frequency and space-time correlations in multiphoton states," Phys. Rev. A, Vol. 64, pp. 063815 (1-7), 2001.

[7] T. S. Humble and W. P. Grice, "Effects of spectral entanglement in polarizationentanglement swapping and type-I fusion gates," Phys. Rev. A, Vol. 77, pp. 022312 (19), 2008.

[8] C. K. Hong, Z. Y. Ou, and L. Mandel, "Measurement of sub-picosecond time intervals between two photons by interference," Phys. Rev. Lett, Vol. 59, pp. 2044-2046, 1987.

[9] M. Chekhova, "Polarization and Spectral Properties of Biphotons," Prog. Opt. Vol. 56, pp. 187-225, 2011.

[10] C. I. Osorio, N. Sangouard, and R. T. Thew, "On the purity and indistinguishability of down-converted photons." J. Phys. B: At. Mol. Opt. Phys, Vol. 46, pp. 055501 (1-11), 2013.

[11] J. Audretsch, Entangled Systems: New Directions in Quantum Physics, John Wiley \& Sons, 2008.

[12] G. B. Arfken and H. J. Weber, Mathematical Methods for Physicists, Academic Press, $6^{\text {th }}$ Ed. 2005.

[13] Z. J. Ou, Multi-photon quantum interference, New York: Springer, 2007.

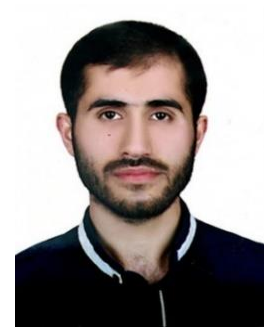

Ali Mohammadi was born in Rasht, Iran, in 1991. He received the B.S. degree in solid state physics from the Shahed University, Tehran, Iran, in 2014, and the M.S. degree in photonics from the Laser and Plasma Research Institute, Shahid Beheshti University, Tehran, Iran, in 2017. 
His research experience include nonlinear optics and quantum optics, specifically study about produces and detects of entangled photons from spontaneous parametric down conversion (SPDC) process. He also presented two papers on the characteristics of entangled photon pairs from SPDC process in 22rd and 23rd iranian conference on optics and photonics (ICOP 2016 and 2017).

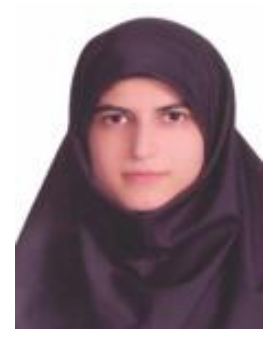

Atoosa Sadat Arabanian was born in Tehran, Iran in 1980. She received the B.S. degree in applied physics from the Azad university of Karaj in 2003, and the M.S. degree in photonics from the Laser and Plasma Research Institute, Shahid Beheshti University, Tehran, Iran, in 2006, and she was earned her $\mathrm{PhD}$ degree in Photonics September 2013 at the Laser and Plasma Research Institute, Shahid Beheshti University, Tehran, Iran.

She is currently an assistant professor at the Laser and Plasma Research Institute of Shahid
Beheshti University. Her research fields include technology of femtosecond fiber lasers and continuous fiber lasers, femtosecond laser micromachining, optical coherent tomography of biological samples and experimental quantum optics.

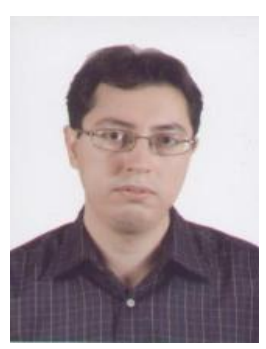

Ali Dalafi received his B.Sc. from Sharif University of Technology, Tehran, Iran, his M.Sc. degree from University of Tehran in condensed matter physics and his Ph.D. degree from University of Isfahan in theoretical quantum optics. He currently works as an assistant professor in the Laser and Plasma Research Institute, Shahid Beheshti University, Tehran. His research interests include theoretical quantum optics, quantum optomechanics, quantum measurement, BoseEinstein condensation and quantum manybody system. 
THIS PAGE IS INTENTIONALLY LEFT BLANK. 